\title{
HIGHLIGHTS
}

PARKINSON DISEASE

\section{Could sunlight offer protection from Parkinson disease?}

Inadequate serum levels of 25-hydroxyvitamin D could be putting individuals at an increased risk of developing Parkinson disease (PD), according to a new study. Epidemiologists at the National Institute for Health and Welfare, Helsinki, Finland suggest that this risk is heightened threefold in individuals with low levels of this hormone, which is obtained through the diet and also through photosynthetic reactions in the skin following ultraviolet B irradiation.

A paper published in 2007 hypothesized that a link might exist between vitamin $\mathrm{D}$ intake and the pathogenesis of PD. In their study, Paul Knekt and colleagues investigated whether a population with low serum vitamin D levels related to limited sun exposure have an elevated risk of developing PD later in life.

From the participants in a survey conducted in Finland between 1978 and 1980, Knekt et al. selected a cohort of 3,173 men and women aged $50-79$ years, none of whom had PD at baseline. Using blood samples obtained at the time of the initial survey, the researchers measured the vitamin D status of the cohort. The team discovered, after adjusting for several possible confounders (BMI, physical activity, alcohol and smoking, education, marital status, and time of year of blood test), that higher serum vitamin D levels (at least $50 \mathrm{nmol} / \mathrm{l})$ predicted a lower risk $(65 \%$ lower than in individuals with serum levels of $<25 \mathrm{nmol} / \mathrm{l}$ ) of developing PD.

The research, which had a 29 year follow-up period, is, according to Knekt, "the first study to provide human data in a longitudinal study design to suggest that inadequate vitamin D status is associated with the risk of developing PD."

This study now needs to be followed up with cohorts of people from different latitudes to determine whether low levels of this hormone, resulting from limited sun exposure and low vitamin D intake, can accurately predict $\mathrm{PD}$, since the risk factors

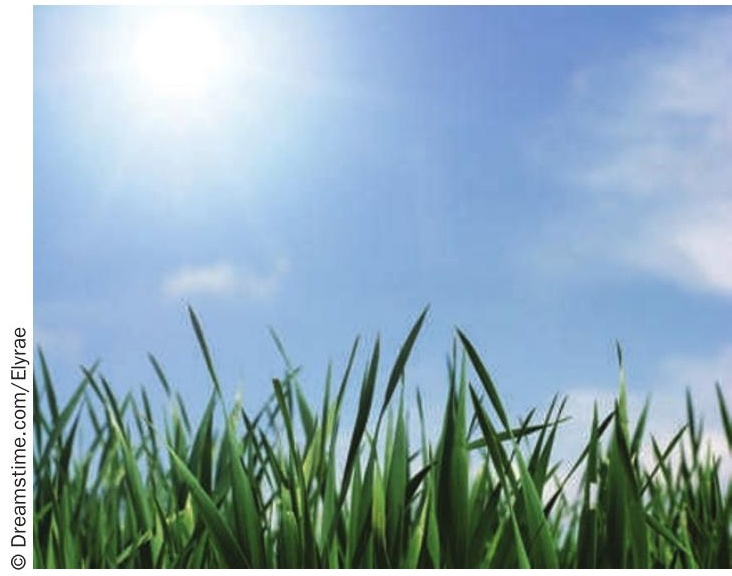

of this disease are not very well known. Although the neuroprotective effects of vitamin D have been previously shown, it is now necessary to "elucidate the exact role, mechanisms and optimum concentrations of vitamin D in PD," explains Knekt, adding that "in clinical trials focusing on the effect of vitamin D supplements, the incidence of PD merit follow-up."

Carolyn McSharry

Original article Knekt, P. et al. Serum vitamin D and the risk of Parkinson disease. Arch. Neurol. 67, 808-811 (2010) 